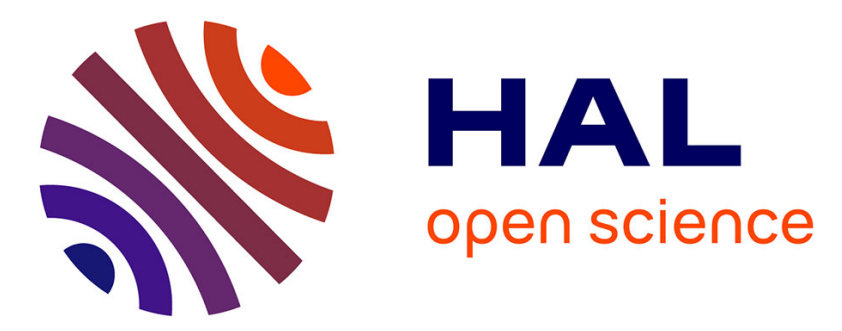

\title{
Maintenance of handedness polymorphism in humans: A frequency-dependent selection model
}

\author{
Sylvain Billiard, Charlotte Faurie, Michel Raymond
}

\section{To cite this version:}

Sylvain Billiard, Charlotte Faurie, Michel Raymond. Maintenance of handedness polymorphism in humans: A frequency-dependent selection model. Journal of Theoretical Biology, 2005, 235, pp.85-93. 10.1016/j.jtbi.2004.12.021 . halsde-00184539

\section{HAL Id: halsde-00184539 \\ https://hal.science/halsde-00184539}

Submitted on 31 Oct 2007

HAL is a multi-disciplinary open access archive for the deposit and dissemination of scientific research documents, whether they are published or not. The documents may come from teaching and research institutions in France or abroad, or from public or private research centers.
L'archive ouverte pluridisciplinaire HAL, est destinée au dépôt et à la diffusion de documents scientifiques de niveau recherche, publiés ou non, émanant des établissements d'enseignement et de recherche français ou étrangers, des laboratoires publics ou privés. 


\title{
Maintenance of handedness polymorphism in humans:
}

\section{A frequency-dependent selection model $^{1}$}

\author{
Sylvain Billiard ${ }^{1}$, Charlotte Faurie ${ }^{1, *}$ and Michel Raymond ${ }^{1}$ \\ ${ }^{1}$ The authors contributed equally and are listed in alphabetical order. \\ ${ }^{1}$ Institute of Evolutionary Sciences of Montpellier, France. \\ ${ }^{*}$ Corresponding author. Institute of Evolutionary Sciences \\ University of Montpellier II - CC 065 \\ Place Eugène Bataillon \\ F 34095 Montpellier cedex 5 \\ Telephone: +33467144632 \\ Fax: +33467143622 \\ E-mail: faurie@,isem.univ-montp2.fr
}

\begin{abstract}
Frequency-dependent selection is an important process in the maintenance of genetic variation in fitness. In humans, it has been proposed that the polymorphism of handedness is maintained by negative frequency-dependent selection, through a strategic advantage of left-handers in fighting interactions. Using simple mathematical models, we explore: $1^{\circ}$ ) whether it is possible to predict the range of left-handedness frequencies observed in human populations by the frequency and the violence of fighting interactions; $2^{\circ}$ ) the consequences of the sex differences in the probability of transmission of hand preference to offspring. We show that a wide range of values of the frequency of lefthanders can be obtained with realistic changes of the parameters values. Our models reinforce the idea that negative frequency dependence may have played a role in maintaining left-handedness in human populations, and provide further support for the importance of fighting interactions in the evolution of hand preference. Moreover, they suggest an explanation for the occurrence of left-handedness among women in this context, namely an indirect selective advantage through their male offspring.
\end{abstract}

Keywords: Human evolution, polymorphism, frequency-dependence, handedness.

\footnotetext{
${ }^{1}$ This article was published in Journal of Theoretical Biology (235) Billiard S., Faurie, C., Raymond M., Maintenance of handedness polymorphism in humans: a frequency-dependent selection model, Pages 8593, (C) 2005, and is posted with permission from Elsevier. JOURNAL OF THEORETICAL BIOLOGY Home Page: http://www.ScienceDirect.com/science/journal/00225193
} 


\section{Introduction}

A polymorphism for a non-neutral trait can be observed in a population when there is a balance of selective forces. This can occur either if this trait is under frequency-dependent selection, or if there is a spatial or temporal heterogeneity of selective pressures (Maynard Smith, 1989). If different values of the trait are associated to a frequency-dependent selective cost, or advantage, then stable coexistence will result. The most widespread and dramatic genetic polymorphism, that of sexual dimorphism, is certainly maintained by negative frequency-dependent selection (Fisher, 1958). Negative frequency-dependent selection is a potentially important process in the maintenance of genetic variation in fitness traits, as has been described for the maintenance of the polymorphism of courtship in Drosophila (Ayala and Campbell, 1974), color morphs in lizards, fishes and plants (Endler, 1988; Gigord, Macnair and Smithson, 2001; Sinervo and Lively, 1996), mouth morphology in scale-eating fishes (Hori, 1993), bill crossing morphs in crossbills (Benkman, 1996), cytoplasmic male-sterility factors in gynodioecious plant (Städler and Delph, 2002), major histocompatibility complex in mammals (Meyer and Thomson, 2001), etc.

In humans, handedness is one of the traits for which the maintenance of a polymorphism is probably due to negative frequency-dependent selection (Vallortigara and Rogers, in press). Hand preference is heritable (see e.g. Francks et al., 2002; McKeever, 2000; McManus, 1991; Sicotte, Woods and Mazziotta, 1999), and a handedness polymorphism is detected in early human populations (Faurie and Raymond, 2004) and observed in all contemporary populations as well (Faurie et al., 2005; Raymond and Pontier, 2004). However, left-handedness seems to be associated with several fitness costs, such as a lower height or a reduced longevity (e.g. Aggleton, Kentridge and Neave, 1993; Coren and Halpern, 1991; Gangestad and Yeo, 1997; McManus and Bryden, 1991). The costs reported in the literature are not likely to be frequency-dependent. A frequencydependent advantage is therefore required to explain the maintenance of the polymorphism. (Raymond et al., 1996) have proposed a negative frequency-dependent selection mechanism related to fighting interactions. As left-handers are less frequent, one is more likely to be confronted with a right-handed opponent in a physical fight. Left-handers would thus be more accustomed to righthanded competitors than vice versa. Therefore, they might enjoy a negatively frequency-dependent strategic advantage in fights when rare, relative to right-handers. This frequency-dependent superiority of left-handers in interactive contests would confer them fitness advantages, directly and indirectly. It could have historically influenced survival, but also social status and reproductive success (see, e.g., Archer, Holloway and McLoughlin, 1995; Chagnon, 1988; Hill, 1984).

The action of a negative frequency-dependent advantage of left-handers in physical fights is strongly suggested by the study of interactive sports, which can be considered as a form of fighting interaction. Indeed, left-handers are significantly more frequent among competitors in these sports than in the general population or among non-interactive sport competitors (Aggleton and Wood, 1990; Raymond et al., 1996; Wood and Aggleton, 1989). In sports where the interaction is direct, the frequency of left-handers is almost reaching one half, and it is lower when the interaction is less direct (Grouios et al., 1999). Game-theoretic modelling of handedness in both batting and pitching in baseball has found that models incorporating frequency dependence provide a good fit to historical data on handedness (Goldstein and Young, 1996). Similarly, in cricket, left-handed batsmen have a strategic advantage that decreases as left-handers become more common in competition, which is consistent with frequency-dependent rather than uniform benefits of lefthandedness in interactive contests (Brooks et al., 2003). Another empirical support of the fighting hypothesis is the cross-cultural positive correlation found in traditional societies between the rate of homicides and the frequency of left-handedness (Faurie and Raymond, 2005). This correlation is predicted by the fighting hypothesis, as an increased level of violence (and thus of dual fights) provides a greater fitness advantage to left-handers, which thus increase in frequency. Physical fights could therefore be involved in the selection pressures acting on the frequency of left-handers in human populations. This hypothesis is essentially developed as a verbal argument, and does not formally consider inheritance processes, sex differences, and frequency dependence. 
The primary aim of the article was to determine the range of parameters values to consider to be able to predict the whole range of the left-handedness frequencies observed in human populations. Furthermore, there is a problematic issue in the context of this hypothesis. As male/male aggression in humans is much more frequent than aggression involving females (e.g. Manning and Taylor, 2001; Mesquida and Wiener, 1999), the fighting advantage should concern mostly left-handed men. Considering that the costs apply to both sexes, the mere existence of lefthanded women is puzzling. Nevertheless, the frequency of left-handers among women is close to the frequency among men (although generally lower) (Annett, 1985; Porac and Coren, 1981). We investigated whether the probabilities of transmission of hand preference to offspring could reflect the proximal mechanism for this phenomenon. Indeed, the probability for a child to be left-handed increases when one of his/her parents is left-handed and, more remarkably, this increase is higher when the mother is left-handed than when the father is left-handed (e.g. McKeever, 2000; McManus, 1991; Porac and Coren, 1981). There are thus stronger maternal effects than paternal effects upon offspring handedness. Such a finding could result from a sex-related genetic effect, or from a greater social influence on the child likely to be exerted by the mother.

In the first section of this article, a simple mathematical model of frequency-dependent selection is presented; it serves as a basis for the models of the following sections. In the second section, we investigate whether the frequency of left-handers at equilibrium in a population may be predicted by the frequency and the violence of fighting interactions. We study the influence of different parameters, representing the underlying mechanisms of the frequency-dependent advantage in fighting. These parameters are: probability of fighting during an individual's life, probability of death during a fight, cost of being left-handed, and the frequency-dependent advantage of left-handers in fights. We also consider in this section the influence of the costs and advantages associated to involvement in fights. In the third section, we investigate the consequences of the sex differences in the probability of transmission of hand preference to offspring. All computations were performed using Mathematica version 4 (Wolfram Research, Inc. 1988-1999).

\section{The basic negative frequency-dependent selection model of the evolution of hand preference}

In this section, we present the frequency-dependent selection model that we will use throughout this paper. We suppose an infinite population, so that we neglect stochastic changes of the phenotypes frequency, and we study the fitness of individuals depending on their hand preference. We respectively note $f_{L}(t)$ and $f_{\mathrm{R}}(t)$ the frequency of left- and right-handed individuals in the population at generation $t$. At any time we have $f_{L}(t)=1-f_{R}(t)$. We assume that left-handers pay a cost of fitness $c$ and that they have a frequency-dependent advantage $\Delta$ of the form $\Delta \equiv \delta\left(1 / 2-f_{L}(t)\right) / f_{L}(t)$ (Hedrick, 1985). Under this assumption, $\Delta$ is positive while left-handers are less than $1 / 2$. Moreover, $\Delta$ is very large when left-handers are very rare, as it is expected to be in natural populations. Finally, this form of frequency-dependent selection is easily tractable in computations and allows finding frequencies at equilibrium without many approximations. The advantage is modulated by $\delta$, a positive constant.

The aim of this model is to compute the frequency of left-handers at equilibrium. Under our assumptions in this section, the fitness of left-handers at generation $t$ is $W_{L}(t)=(1+\Delta)(1-c)$, while the fitness of right-handers is $W_{R}(t)=1$. The frequency of left-handed individuals at generation $t+1$ is therefore:

$$
\begin{aligned}
& f_{L}(t+1)=\frac{f_{L}(t) W_{L}(t)}{f_{L}(t) W_{L}(t)+f_{R}(t) W_{R}(t)} . \\
& f_{L}^{\circ}=\frac{(1-c) \delta}{2 \delta+2 c(1-\delta)} .
\end{aligned}
$$


We see in equation (2) that the frequency of left-handers tends towards 0 when $c$ goes to 1 and that $\lim _{\delta \rightarrow \infty} f_{L}^{\circ}=1 / 2$. As shown on Figure 1 , the frequency reaches high values for a small $\delta$. The range of values found in the literature for the frequency of left-handedness across human populations is reported also in Figure 1. No estimation of the global cost of left-handers is available in the literature, but whatever the value of $c$ chosen here, the model can predict the whole range of frequencies. Moreover, under this kind of selection, relatively small variations in the value of the advantage are sufficient to explain the observed variations in the prevalence of left-handedness. In other words, it is not necessary that the advantage of left-handers be large to obtain high frequencies at equilibrium.

\section{Effects of fights on the frequency of left-handed males at equilibrium}

In this section, we use a specific mechanism to describe the frequency-dependent advantage of left-handed males in fights. Hence, we only consider males' handedness. Fitness is assumed to be the probability of survival until age of maturity, and depends on hand preference. The survival probability is decomposed into two components: the probability to be killed in fights and the probability to die before reproduction because of intrinsic reasons (this second component is introduced to take into account the cost of left-handers). Therefore, if we note $k$ the probability to be killed in fights, and $c$ the probability for left-handers to die before reproduction for intrinsic reasons, the total survival probability of left-handers is $(1-k)(1-c)$.

We suppose that each male has a probability $P_{\text {fght }}$ to be involved in a fight during his life. When a fight occurs between two right-handed males, each male has a probability $P_{\text {deat }}$ to be killed by the other. When a fight occurs between a right-hander and a left-hander, the right-hander has a probability $P_{\text {death }}(1+\Delta)$ and the left-hander a probability $P_{\text {deat }}(1-\Delta)$ to be killed, because of the fighting advantage of left-handers, who are less frequent than right-handers are. The advantage $\Delta$ is defined as in the first section.

All males who have survived until age of maturity are assumed to have the same probability to reproduce and the same amount of offspring: resources for reproduction, including females, are not limiting. All males die after reproduction. As the population is considered infinite, the occurrence of deaths during fights does not change the frequency of right- and left-handed males within a generation.

Under these assumptions, the probability for a left-handed male to survive until reproduction at generation $\mathrm{t}$ is:

$W_{L}(t)=P_{\text {fight }}\left(f_{R}(t)\left(1-P_{\text {death }}(1-\Delta)\right)+f_{L}(t)\left(1-P_{\text {death }}\right)\right)(1-c)+\left(1-P_{\text {fight }}\right)(1-c)$

and the probability of survival for a right-handed male is:

$W_{R}(t)=P_{\text {fight }}\left(f_{L}(t)\left(1-P_{\text {death }}(1+\Delta)\right)+f_{R}(t)\left(1-P_{\text {death }}\right)\right)+\left(1-P_{\text {fight }}\right)$.

Using equation (1), we get the frequency of left-handers at equilibrium:

$f_{L}^{\circ}=\frac{1}{4}\left(3-\frac{2}{c}-\frac{2(1-V)}{\delta V}+\sqrt{8 \frac{(1-c)}{c}+\left(3-\frac{2}{c}-\frac{2(1-V)}{\delta V}\right)^{2}}\right)$ with $V \equiv P_{\text {fight }} P_{\text {death }}$.

The frequency of left-handers at equilibrium is 0 when $V=0$ and $1 / 2$ when $c=0$, as expected. When $c$ and $\delta$ are small, say $c$ and $\delta$ are of the same order as a small parameter $\varepsilon$, we have $f_{L}^{\circ}=\frac{1}{2}-\frac{c(1-V)}{2 \delta V+2 c(1-V)}+\mathrm{O}(\varepsilon)$ (where $O(\varepsilon)$ represents a term of the same order as $\varepsilon$ ), which shows that the frequency at equilibrium increases with violence. A remarkably large range of frequencies of left-handers can be obtained with small variations of the parameters (see Figure 2). For example, with $c=0.1$, i.e. the survival of left-handed males until age of reproduction is 
decreased by a factor $10 \%$ (due to the intrinsic cost of left-handedness), and $\delta=0.05$, which leads to a very low advantage in fights, the frequency of left-handers varies between $f_{L}^{\circ}=0.01$ for $V \approx 0.043$ and $f_{L}^{\circ}=0.1$ for $V \approx 0.355$. It is important to note that the form of the curve depends mostly on the value of the modulator of the advantage $\delta$ (see Figure 2).

$V$, the product of the probabilities of fighting and of dying during a fight, is a measure of the violence prevailing in the population. It is the individual's lifetime probability to die in a fight. In the literature, one of the most frequent index is the homicide rate of the population, which is the number of homicides / 1,000 individuals / year. Homicide rates estimates vary culturally from 0.1 to 10 (Kelly, 1993; Knauft, 1987). Assuming that life expectancy in our model equals 60 years, the corresponding values for $V$ are between 0.006 and 0.6 . The corresponding frequencies of lefthanded males at equilibrium would be between 0.0027 and 0.29 for $\delta=0.1$, which is approximately comparable to the estimated frequencies of left-handers in human populations, ranging from $3 \%$ to $27 \%$ as shown on Figure 1 (Faurie et al., 2005).

We now furthermore consider that fighting itself has specific costs and advantages. Fighting has a cost for every male, because it uses his resources at the expense of reproduction. Therefore, when a male is involved in a fight, his fitness will decrease by a factor $c_{\text {fght }}$. However, fighting may also have social advantages: to be involved in a fight may confer a better status and imply an increase in fitness. Moreover, winning a fight may give a higher increase in fitness because mates may prefer winners. Therefore, we assume in the model that males involved in fights have a fitness increase $s_{\text {fight }}$ and winners have an extra fitness increase $s_{w}$. Left-handed individuals still have an intrinsic cost $c$. When a left-hander fights against a right-hander, he has a probability (1-P death) $(1-$ $\Delta) P_{\text {death }}(1+\Delta)$ to survive and to kill his opponent and then to get an fitness increase $s_{m}$. Using the same notation as before, the fitness of left-handed males at generation $t$ is:

$$
\begin{aligned}
& W_{L}(t)=P_{\text {fight }}\left(f_{R}(t)\left(1-P_{\text {death }}(1-\Delta)\right) P_{\text {death }}(1+\Delta)+f_{L}(t) P_{\text {death }}\left(1-P_{\text {death }}\right)\right)\left(1-C_{\text {fight }}\right)(1-c)\left(1+S_{\text {fight }}\right)\left(1+S_{w}\right) \\
& +P_{\text {fight }} \quad\left(f_{R}(t)\left(1-P_{\text {death }}(1-\Delta)\right)\left(1-P_{\text {death }}(1+\Delta)\right)+f_{L}(t)\left(1-P_{\text {death }}\right)\left(1-P_{\text {death }}\right)\right)\left(1-c_{\text {fight }}\right)(1-c)\left(1+S_{\text {fight }}\right) \\
& +\left(1-P_{\text {fight }}\right)(1-c)
\end{aligned}
$$

and for right-handed males:

$$
\begin{aligned}
& W_{R}(t)=P_{\text {fight }}\left(f_{L}(t)\left(1-P_{\text {death }}(1+\Delta)\right) P_{\text {death }}(1-\Delta)+f_{R}(t) P_{\text {death }}\left(1-P_{\text {death }}\right)\right)\left(1-c_{\text {fight }}\right)\left(1+s_{\text {fight }}\right)\left(1+s_{w}\right) \\
& +P_{\text {fight }}\left(f_{L}(t)\left(1-P_{\text {death }}(1+\Delta)\right)\left(1-P_{\text {death }}(1-\Delta)\right)+f_{R}(t)\left(1-P_{\text {death }}\right)\left(1-P_{\text {death }}\right)\right)\left(1-c_{\text {fight }}\right)\left(1+s_{\text {fight }}\right)+\left(1-P_{\text {fight }}\right)
\end{aligned}
$$

Using equations (1), (6) and (7), we can compute the change in frequency of left-handed males in a generation, assuming that $\delta$ is of order $\varepsilon$ (for clarity we have noted the left-handed frequency at generation $t$ simply $f_{L}$ in the right part of the following equation):

$$
f_{L}(t+1)-f_{L}(t)=\frac{1-f_{L}}{2\left(1-c f_{L}\right)^{2}}\left[-2 c f_{L}\left(1-c f_{L}\right)+\frac{A_{1}\left(1-2 f_{L}\right)\left(1+(1-c) f_{L}\right) V \delta}{\left(1+f_{L}\right)\left(1-A_{2} P_{\text {fight }}\right)}\right]+o(\varepsilon)
$$

$$
\begin{array}{r}
\text { where } A_{1} \equiv(1-c)\left(1-c_{\text {fight }}\right)\left(1+s_{\text {fight }}\right)\left(1+s_{w}\right) \text { and } \\
A_{2} \equiv 1-\left(1-c_{\text {fight }}\right)\left(1+s_{\text {fight }}\right)\left(1-P_{\text {death }}\left(1-s_{w}\left(1-P_{\text {death }}\right)\right)\right)
\end{array}
$$

It is not possible to find exact equilibrium values of left-handedness frequency using equation (8). We have therefore determined equilibrium values in a numerical way with recursive 
equations (6) and (7); these are shown on Figure 3. We can see on this figure that a social advantage associated to fighting significantly increases the frequency of left-handers in the population at equilibrium. We also observe (thick line) how the frequency of left-handers decreases when the cost of fighting increases. Right- and left-handers have the same social advantage associated to fighting, $s_{\text {figh }}$, and the same probability to be engaged in fight, however we can see on Figure 3 that the frequency of left-handers increases with $s_{f g h b}$, due to their smaller overall probability of dying during a fight, as compared to right-handers.

\section{Why do left-handed females exist? An indirect advantage hypothesis.}

In human populations, the frequency of left-handers among women is generally slightly lower than among men (e.g. Annett, 1985; Porac and Coren, 1981). In this section, we try to understand how left-handedness can be present in females despite the absence of any direct advantage, in the context of the hypothesis of an advantage in fights for males. We propose a model taking into account the probability of transmission of the hand preference character, given the hand preference of both parents. In this model, we will consider that the sex ratio is fixed and equal to $1 / 2$, at any generation and at each step of the life cycle (populations are infinite). There is no sexual preference for mate's handedness: reproduction occurs between randomly chosen males and females. In this model, we do not consider a specific mechanism for the advantage of left-handed males. We simply assume that left-handed males have a frequency-dependent advantage in fitness (through their survival probability), as in the first section: $\Delta \equiv \delta\left(1 / 2-f_{L}(t)\right) / f_{L}(t)$. Although this advantage concerns only males, both sexes pay a cost for being left-handed, respectively $c_{M}$ and $c_{\mathrm{F}}$ for males and females.

We introduce now the following notation: $M_{X}(t)$ and $F_{X}(t)$ are respectively the frequency of males and females with hand preference $\mathrm{X}$ in the whole population at generation $t, \mathrm{X}$ taking the value $\mathrm{R}$ for right-handers and $\mathrm{L}$ for left-handers.

Note that $M_{L}+M_{R}+F_{L}+F_{R}=1$, and since the sex ratio is assumed $1 / 2$, $M_{L}+M_{R}=F_{L}+F_{R}=1 / 2$. Thus the frequency of $X$ males among males alone is $2 M_{X}$.

We note $p_{X Y}$ (respectively $q_{X Y}$ ) the probability for a male $\mathrm{X}$ and a female $\mathrm{Y}$ to have a lefthanded offspring, given that this offspring is a male (respectively a female). The life cycle is composed of the following steps: first, reproduction occurs between randomly chosen males and females, the hand preference of their offspring depending on $p_{X Y}$ and $q_{X Y}$. We will refer to this step as "transmission" and use the superscript " $\mathrm{T}$ " for the frequencies of the different phenotypes in the population after this step. All adults die and selection on the offspring occurs: left-handed individuals pay an intrinsic cost $c_{\mathrm{F}}$ and $c_{M}$, but left-handed males have an advantage $\Delta$.

\section{First step: frequencies after transmission.}

The frequency of XY couples is $4 M_{X} F_{Y}$. The frequency of males among their offspring is $1 / 2$. Frequencies after transmission can then be written in matrix form:

$$
\left(\begin{array}{c}
M_{L}^{T}(t) \\
M_{R}^{T}(t) \\
F_{L}^{T}(t) \\
F_{R}^{T}(t)
\end{array}\right)=2\left(\begin{array}{cccc}
p_{\mathrm{LL}} & p_{\mathrm{RL}} & p_{\mathrm{LR}} & p_{\mathrm{RR}} \\
1-p_{\mathrm{LL}} & 1-p_{\mathrm{RL}} & 1-p_{\mathrm{LR}} & 1-p_{\mathrm{RR}} \\
q_{\mathrm{LL}} & q_{\mathrm{RL}} & q_{\mathrm{LR}} & q_{\mathrm{RR}} \\
1-q_{\mathrm{LL}} & 1-q_{\mathrm{RL}} & 1-q_{\mathrm{LR}} & 1-q_{\mathrm{RR}}
\end{array}\right) \cdot\left(\begin{array}{l}
M_{L}(t) F_{L}(t) \\
M_{R}(t) F_{L}(t) \\
M_{L}(t) F_{R}(t) \\
M_{R}(t) F_{R}(t)
\end{array}\right)
$$

In accordance with literature data on transmission of hand preference (e.g. McKeever, 2000; Porac and Coren, 1981), we assume that an individual offspring has a higher probability to be left-handed if one of the parents is left-handed, and even higher if both parents are left-handed. To reduce the number of parameters in the model, we use the following parameters: $p$ and $q$ are the probabilities that two right-handed parents have a left-handed offspring, given that the offspring is 
a male or a female, respectively. If the father is left-handed then a male (resp. a female) offspring has a probability to be also left-handed increased by a factor $\alpha_{\mathrm{M}}$ (resp. $\alpha_{\mathrm{F}}$ ). If the mother is lefthanded, the probability of being left-handed for a male (resp. female) is increased by a factor $\beta_{\mathrm{M}}$ (resp. $\beta_{\mathrm{F}}$ ). We can now express the elements in the transmission matrix of equation (9) as follows:

$$
\begin{aligned}
& p_{L L}=p\left(1+\alpha_{M}\right)\left(1+\alpha_{F}\right) ; \quad p_{R L}=p\left(1+\alpha_{F}\right) ; \quad p_{L R}=p\left(1+\alpha_{M}\right) ; \quad p_{R R}=p ; \\
& q_{L L}=q\left(1+\beta_{M}\right)\left(1+\beta_{F}\right) ; \quad q_{R L}=q\left(1+\beta_{F}\right) ; \quad q_{L R}=q\left(1+\beta_{M}\right) ; \quad q_{R R}=q \text {. }
\end{aligned}
$$

\section{Second step: frequencies after selection.}

Using the same kind of equations as in the first section to compute the frequency changes for both phenotypes, we obtain:

$$
\begin{aligned}
M_{L}(t+1) & =\frac{\left(1-c_{M}\right)\left(1+\delta\left(1 / 2-2 M_{L}^{T}(t)\right) /\left(2 M_{L}(t)\right)\right)}{\left(1-c_{M}\right)\left(1+\delta\left(1 / 2-2 M_{L}^{T}(t)\right) /\left(2 M_{L}(t)\right)\right)+2 M_{R}(t)} M_{L}(t) \\
M_{R}(t+1) & =\frac{1}{\left(1-c_{M}\right)\left(1+\delta\left(1 / 2-2 M_{L}^{T}(t)\right) /\left(2 M_{L}(t)\right)\right)+2 M_{R}(t)} M_{R}(t) \\
F_{L}(t+1) & =\frac{\left(1-c_{F}\right)}{\left(1-c_{F}\right) 2 F_{L}^{T}(t)+2 F_{R}^{T}(t)} F_{L}^{T}(t) \\
F_{R}(t+1) & =\frac{1}{\left(1-c_{F}\right) 2 F_{L}^{T}(t)+2 F_{R}^{T}(t)} F_{R}^{T}(t)
\end{aligned}
$$

Third step: frequencies at equilibrium considering transmission and selection.

solve:

To compute the frequency of left-handed males and females at equilibrium, we have to

$$
\begin{gathered}
M_{L}(t+1)-M_{L}(t)=0 \\
F_{L}(t+1)-F_{L}(t)=0
\end{gathered}
$$

We assume that that $\alpha_{\mathrm{F}}, \alpha_{\mathrm{M}}, \beta_{\mathrm{F}}$ and $\beta_{\mathrm{M}}$ are all small and of the same order as $\varepsilon$, that is to say that left-handers have a slightly increased probability to produce left-handed offspring relatively to right-handers. We can then compute frequencies at equilibrium by solving:

$$
\begin{aligned}
& Q_{M}(t)=0 \quad \begin{aligned}
M_{L}(t+1)-M_{L}(t) & =Q_{M}(t)+o(\varepsilon) \\
Q_{F}(t) & =0
\end{aligned} \text { with } \quad F_{L}(t+1)-F_{L}(t)=Q_{F}(t)+o(\varepsilon)
\end{aligned} .
$$

On Figure 4, we show the effect of the cost on the frequency at equilibrium for both sexes. The intersection between thin lines and y-axis gives the equilibrium values without any selection (no cost and no advantage). In absence of selection, left-handedness frequency at equilibrium is the result of transmission only, and left-handed males and females have the same frequency at equilibrium. On this figure, we can see that under these assumptions and parameters values, the advantage of left-handed males has very little influence on the frequency of left-handed females at equilibrium. However, as shown on Figure 5, the frequency of left-handed females increases more with the advantage when the probability of transmission is higher for females. Therefore, even if the advantage of being left-handed is expressed in males only, left-handed females are indirectly favoured if they have a higher probability to have left-handed sons, as reported in the literature. Is is important to notice that in our model, we have not specified how left-handedness is transmitted. Transmission may be either cultural, genetic or both. Hence, if a part of the inheritance is due to cultural transmission, we can explain why females have to express left-handedness even if they do not have any direct advantage. 


\section{Discussion}

Polymorphic traits under frequency-dependent are likely to be observed more frequently than polymorphic traits under directional selection, because in the latter case one phenotype is rapidly fixed. Therefore, it is expected that many of human heritable traits being polymorphic in current populations are the result of balancing selection, which is most often frequency-dependent. The result is usually a $50 \%$ polymorphism, when two phenotypes are involved and when frequencydependence alone is acting. Concerning handedness, the fact that there is a sex-specific advantage, and a cost in addition, yields to a more complex system. Our models have highlighted the pertinence of frequency-dependent selection as an explanation for the persistence of the polymorphism of handedness.

In the first model, we have shown that a wide range of values of the frequency of lefthanders can be obtained with reasonable changes of the parameters values. In particular, a slight negative frequency-dependent advantage of left-handed individuals is sufficient to countervail a fitness cost. These results reinforce the hypothesis that negative frequency dependence may have played a role in maintaining left-handedness in human populations.

In the second section, we have analysed possible mechanisms for a frequency-dependent advantage of left-handed males in fighting interactions and its fitness consequences. Our results provide further support for the importance of fighting interactions in the evolution of hand preference. The frequency of left-handers is highly variable between human populations (Faurie et al., 2005; Raymond and Pontier, 2004). Fighting interactions could be involved in this variability. The frequency of left-handers is expected to be higher in societies where physical fights are frequent and violent. Indeed, the frequency of left-handers has been shown to be positively correlated with the rate of homicides in traditional societies (Faurie and Raymond, 2005). The frequency-dependent advantage of a rare phenotype in aggressive interactions is not likely to be restricted to humans. It has been described for example in predator-prey interactions (Vallortigara and Rogers, in press). It would be informative to investigate it in intraspecific fights among nonhuman primates. In capuchin monkeys, the rate of killing is estimated to 8/ 1000 adults /year (Gros-Louis, Perry and Manson, 2003), which is in the range of the most violent human societies, and the frequency of left-handed throwers is slightly below 50\% (Cleveland et al., 2003; Westergaard et al., 2000). In chimpanzees' populations, the rate of killing is approximately the same (Wrangham, 1999), and it seems that the frequency of left-handed throwers is relatively high as well (around 30\%) (Hopkins et al., 1993; McGrew and Marchant, 1997). These fragmentary data suggest that the costs associated to left-handedness are present in chimpanzees but absent or very low in capuchin monkeys, as the frequency of left-handers is expected to be 0.5 without a fitness cost. This opens new and interesting perspectives for the evaluation of the nature and the significance of these costs. Tentatively, it would be interesting to study the correlation between mortality rates in fights and a measure of handedness across primates' species.

In the last model, we have shown that, despite the costs carried by both males and females, left-handed females can benefit of the advantage of their sons who are more likely to be lefthanded. This represents an indirect advantage for left-handed women, and it could explain how they are maintained. If the transmission of left-handedness were strictly genetic, the phenotypic expression of the "left-handed genes" would be expected to decrease in women, because of the associated cost. However, the phenotype itself is probably involved in the transmission as well, e.g. through imitation (Laland et al., 1995). Therefore, women would still express left-handedness to confer to their sons the advantage in fighting interactions.

Alternatively, left-handedness could be associated with other advantages, either femalespecific or not. Indeed, the existence of some unknown advantages of left-handedness is not excluded. For example, the advantage in interactive sports could have some importance in Western societies, where it has been shown that student athletes have a higher number of sexual partners (Faurie, Pontier and Raymond, 2004). If there are no fitness-related advantages of left-handedness in Western societies, left-handedness may disappear at equilibrium, except if, for example, the improvement of medical care leads to a suppression of the costs. 
In our models, fitness costs associated to left-handedness are fixed parameters. However, an alternative explanation for the variability of the frequency could be that the intensity of the costs varies across populations. The costs have only been studied in Western societies. Their existence in traditional societies can be inferred from the existence of the frequency-dependent advantage in fighting interactions (suggested by the correlation observed between the frequency of left-handers and the rate of homicides, Faurie and Raymond, 2005): in absence of any cost, such an advantage would lead to a frequency of $50 \%$ at equilibrium. The potential variability of the costs across human populations has never been investigated.

The models described in the present article cannot be applied to sportsmen and athletes, because they do not constitute a population (in the reproductive meaning). The overrepresentation of left-handers in interactive sports reflects their frequency-dependent advantage alone, apart from the intrinsic cost, the probability of survival, etc. This is why we observe, among champions in certain sports which are very close to real fighting interactions, frequencies almost reaching $50 \%$, the maximum possible frequency in the context of the frequency-dependent advantage (Brooks et al., 2003; Goldstein and Young, 1996; Grouios et al., 2000; Raymond et al., 1996). The fact that these values are never found in human populations, even in the populations known to be the most violent, indicates that the cost associated to left-handedness is relatively high (Faurie and Raymond, 2005).

The widespread existence of the costs across human populations seems to weaken the hypotheses classically considered in Western societies. A widespread statement is that the costs are mainly due to the presence of numerous asymmetrical artefacts conceived to be handled by righthanders (Aggleton, Kentridge and Neave, 1993; Coren, 1989; Daniel and Yeo, 1994; Porac and Coren, 1981) For example professional tools like saws can be very dangerous for a left-handed user. In traditional societies, asymmetrical tools are rather uncommon. Moreover, most tools are personal possessions, and then adapted to the owner's hand preference. This is illustrated among the Ntumu of Cameroon, where the machete sharpened for either left- or right-hand use is named differently. Each individual, including women and children from age 5, has a personal machete sharpened according to his/her own hand preference (Carrière and Raymond, 2000). The inventory of tools and weapons that lead to different risk levels whether they are used by a left- or a righthander needs to be done in traditional societies. On the basis of the current knowledge, it doesn't seem reasonable to state that the nature of the cost proposed for Western societies applies to traditional societies.

If a ubiquitous cost of left-handedness exists, it is more probably a developmental cost, as there are developmental differences in the brain of left- and right-handers. Under natural selection pressures, the cost associated to a new phenotype is expected to be compensated progressively, for example by the apparition of modifier genes (McKenzie and Batterham, 1994). The polymorphism of handedness is present at least since the upper Palaeolithic (30,000 BP) (Faurie and Raymond, 2004), and probably since much longer, since data indicate that it is present in chimpanzees as well (Hopkins and Pearson, 2000; McGrew and Marchant, 1997). 30,000 years corresponds to about 1,200 generations of 25 years, which is plenty enough for genetic innovations involving a small number of genes to rise, as shown by the specific adaptations to the new lifestyle appeared since the Neolithic (10,000 BP), for example lactose tolerance (McCracken, 1971). This suggests that the cost associated to left-handedness cannot be decreased by a simple accumulation of mutations with additive effects. The constraint involved, which remains to be identified, is most probably associated to functions with a complex genetic determinism, like brain organisation.

The fitness-related characteristics of left- and right-handers need to be further investigated, especially comparing both sexes and different human populations, in order to be able to fully understand the dynamics of this polymorphism.

\section{Acknowlegments}

Many thanks to Georges Kunstler, Thomas Lenormand and Anders P. Møller for valuable suggestions, discussions and improvements, and to Valérie Durand for bibliographic help. This is contribution 05.xxx of the Institute of Evolutionary Sciences of Montpellier (UMR CNRS 5554). 


\section{References}

Aggleton, J. P., Kentridge, R. W. and Neave, N. J., 1993. Evidence for longevity differences between left handed and right handed men: an archival study of cricketers. J. Epidemiol. Community Health 47, 206-209.

Aggleton, J. P. and Wood, C. J., 1990. Is there a left-handed advantage in "ballistic" sports? Int. J. Psychol. 21, 46-57.

Annett, M., 1985. Left, right, hand and brain: the Right Shift Theory. London, LEA Publishers.

Archer, J., Holloway, R. and McLoughlin, K., 1995. Self-reported physical aggression among young men. Aggressive behavior 21, 325-342.

Ayala, J. and Campbell, C. A., 1974. Frequency-dependent selection. Ann. Rev. Ecol. Syst. 1974, 115-138.

Benkman, C. W., 1996. Are the ratio of bill crossing morphs in crossbills the result of frequency-dependent selection? Evol. Ecol. 10, 119-126.

Brooks, R., Bussière, L. F., Jennions, M. D. and Hunt, J., 2003. Sinister strategies succeed at the cricket World Cup. Proceedings of the Royal Society of London B (Suppl. 3).

Carrière, S. and Raymond, M., 2000. Handedness and aggressive behavior in a Ntumu village in southern Cameroon. Acta ethol. 2, 111-114.

Chagnon, N. A., 1988. Life histories, blood revenge, and warfare in a tribal population. Science 239, 985-992.

Cleveland, A., Rocca, A. R., Wendt, E. L. and Westergaard, G. C., 2003. Throwing behavior and mass distribution of stone selection in tufted capuchin monkeys (Cebus apella). Am. J. Primatol. 61, 159-172.

Coren, S., 1989. Left-handedness and accident-related injury risk. Am. J. Public Heath 79, 1-2.

Coren, S. and Halpern, D. F., 1991. Left-handedness: a marker for decreased survival fitness. Psychol. Bull. 109(1), 90-106.

Daniel, W. F. and Yeo, R. A., 1994. Accident proneness and handedness. Biol. Psychiatry 35, 499.

Endler, J. A., 1988. Frequency-dependent predation, crypsis and aposematic colouration. Phil. Trans. R. Soc. Lond. B. 319, 505-523.

Faurie, C., Pontier , D. and Raymond, M., 2004. Student athletes claim to have more sexual partners than other students. Evol. Hum. Behav. 25(1), 1-8.

Faurie, C. and Raymond, M., 2004. Handedness frequency over more than 10,000 years. Proc. R. Soc. Lond. B 271(1536), S43-S45.

Faurie, C. and Raymond, M., 2005. Handedness, Homicide and Negative Frequency-Dependent Selection. Proc. R. Soc. Lond. B, in press.

Faurie, C., Schiefenhövel, W., Le Bomin, S., Billiard, S. and Raymond, M., 2005. Variation in the frequency of left-handedness in traditional societies. Curr. Anthrop. 46(1)

Fisher, R. A., 1958. The genetical theory of natural selection. New York, Dover.

Francks, C., Fisher, S. E., MacPhie, I. L., Richardson, A. J., Marlow, A. J., Stein, J. F. and Monaco, A. P., 2002. A genomewide linkage screen for relative hand skill in sibling pairs. Am. J. Hum. Genet. 70, 800-805

Gangestad, S. W. and Yeo, R. A., 1997. Behavioral genetic variation, adaptation and maladaptation: an evolutionary perspective. Trends Cognit. Sc. 1, 103-108.

Gigord, L. D. B., Macnair, M. R. and Smithson, A., 2001. Negative frequency-dependent selection maintains a dramatic flower color polymorphism in the rewardless orchid Dactylorhiza sambucina (L.) Soo. Proc. Natl. Acad. Sci. USA 98, 6253-6255.

Goldstein, S. R. and Young, C. A., 1996. "Evolutionnary" stable strategy of handedness in Major League Baseball. J. Comp. Psychol. 110, 164-169.

Gros-Louis, J., Perry, S. and Manson, J. H., 2003. Violent coalitionary attacks and intraspecific killing in wild white-faced capuchin monkeys (Cebus capucinus). Primates 44(4), 341-346.

Grouios, G., Sakadami, N., Poderi, A. and Alevriadou, A., 1999. Excess of non-right handedness among individual with intellectual disability: experimental evidence and possible explanations. J. Intell. Disab. Research 43, 306-313.

Grouios, G., Tsorbatzoudis, H., Alexandris, K. and Barkoukis, V., 2000. Do left-handed competitors have an innate superiority in sports ? Percept. Mot. Skills 90, 1273-1282.

Hedrick, P. W., 1985. Genetics of populations. Boston, Jones and Bartlett Publishers.

Hill, J., 1984. Prestige and reproductive success in man. Ethol. Sociobiol. 5, 77-95.

Hopkins, W. D., Bard, K. A., Jones, A. and Bales, S. L., 1993. Chimpanzee hand preference in throwing and infant cradling: implications for the origin of human handedness. Curr. Anthrop. 34, 786-790.

Hopkins, W. D. and Pearson, K., 2000. Chimpanzee (Pan troglodytes) handedness: variability across multiple measures of hand use. J. Comp. Psychol. 114(2), 126-135. 
Hori, M., 1993. Frequency-dependent natural selection in the handedness of scale-eating Cichlid fish. Science 260, 216-219.

Kelly, R. C., 1993. Constructing Inequality: The Fabrication of a Hierachy of Virtue among Etoro. Ann Arbor, University of Michigan Press.

Knauft, B. M., 1987. Reconsidering Violence in Simple Human Societies: Homicide among the Gebusi of New Guinea. Curr. Anthrop. 28(4), 457-500.

Laland, K. N., Kumm, J., Van Horn, J. D. and Feldman, M. W., 1995. A gene-culture model of human handedness. Behav. Genet. 25(5), 433-445.

Manning, J. T. and Taylor, R. P., 2001. Second to fourth digit ratio and male ability in sport: implications for sexual selection in humans. Evol. Hum. Behav. 22(2), 61-69.

Maynard Smith, J., 1989. Evolutionary genetics. New York, Oxford University Press.

McCracken, R. D., 1971. Lactose deficiency: an example of dietary evolution. Curr. Anthrop. 12, 479-517.

McGrew, W. C. and Marchant, L. F., 1997. On the other hand: current issues in and meta-analysis of the behavioral laterality of hand function in nonhuman primates. Yearbook of Physical Anthropology 40, 201-232.

McKeever, W. F., 2000. A new family handedness sample with findings consistent with X-linked transmission. Br. J. Psychol. 91(1), 21-39.

McKenzie, J. and Batterham, P., 1994. The genetic, molecular and phenotypic consequences of selection for insecticide resistance. Trends Ecol. Evol. 9, 166-169.

McManus, I. C. (1991). The inheritance of left-handedness. Biological asymmetry and handedness, Ciba Foundation Symposium, London, Wiley.

McManus, I. C. and Bryden, M. P., 1991. Geschwind's theory of cerebral lateralization : developing a formal, causal model. Psychol. Bull. 110(2), 237-253.

Mesquida, C. G. and Wiener, N. I., 1999. Male Age Composition and Severity of Conflicts. Politics and the Life Sciences 18(2), 181-189.

Meyer, D. and Thomson, G., 2001. How selection shapes variation of the human major histocompatibility complex: a review. Ann. Hum. Genet. 65, 1-26.

Porac, C. and Coren, S., 1981. Lateral preferences and human behavior. New York, Springer Verlag.

Raymond, M. and Pontier, D., 2004. Is there geographical variation in human handedness ? Laterality 9(4), 35-52.

Raymond, M., Pontier, D., Dufour, A.-B. and Moller, A. P., 1996. Frequency-dependent maintenance of left handedness in humans. Proc. R. Soc. Lond. B 263, 1627-1633.

Sicotte, N. L., Woods, R. P. and Mazziotta, J. P., 1999. Handedness in Twins: A Meta-analysis. Laterality 4(3), 265-286.

Sinervo, B. and Lively, C. M., 1996. The rock-paper-scissors game and the evolution of alternative male strategies. Nature 380, 240-243.

Städler, T. and Delph, L. F., 2002. Ancient mitochondrial haplotypes and evidence for intragenic recombination in a gynodioecious plant. Proc. Natl. Acad. Sci. USA 99, 11730-11735.

Vallortigara, G. and Rogers, L. J., in press. Survival with an asymmetrical brain: advantages and disadvantages of cerebral lateralization. Behav. Brain Sc.

Westergaard, G. C., Liv, C., Haynie, M. K. and Suomi, S. J., 2000. A comparative study of aimed throwing by monkeys and humans. Neuropsychol. 38, 1511-1517.

Wood, C. J. and Aggleton, J. P., 1989. Handedness in 'fast ball' sports: do left-handers have an innate advantage? Br. J. Psychol. 80, 227-240.

Wrangham, R. W., 1999. The evolution of coalitionary killing. Yearbook of Physical Anthropology 42, 1-30. 
Figure 1. Frequency of left-handers at equilibrium $f_{L}$ as a function of their advantage (see equation 2), for various fitness costs of left-handedness $c$. The area highlighted in grey represents the range of values of the frequency of left-handers so far observed in human populations.

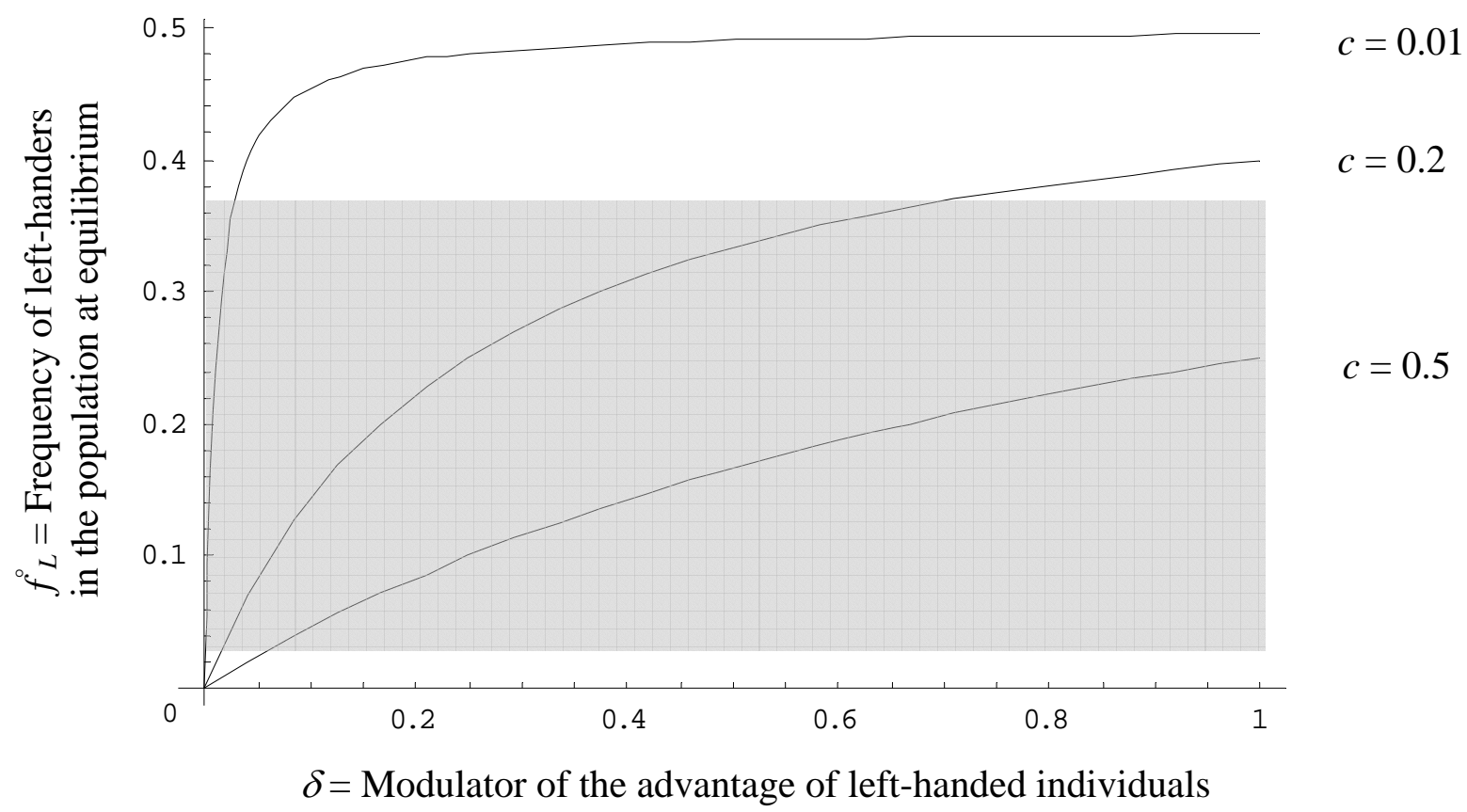

Figure 2. Frequency of left-handers at equilibrium as a function of the level of violence in the population (probability of death due to fighting within a generation). For all curves $c=$ 0.1 .

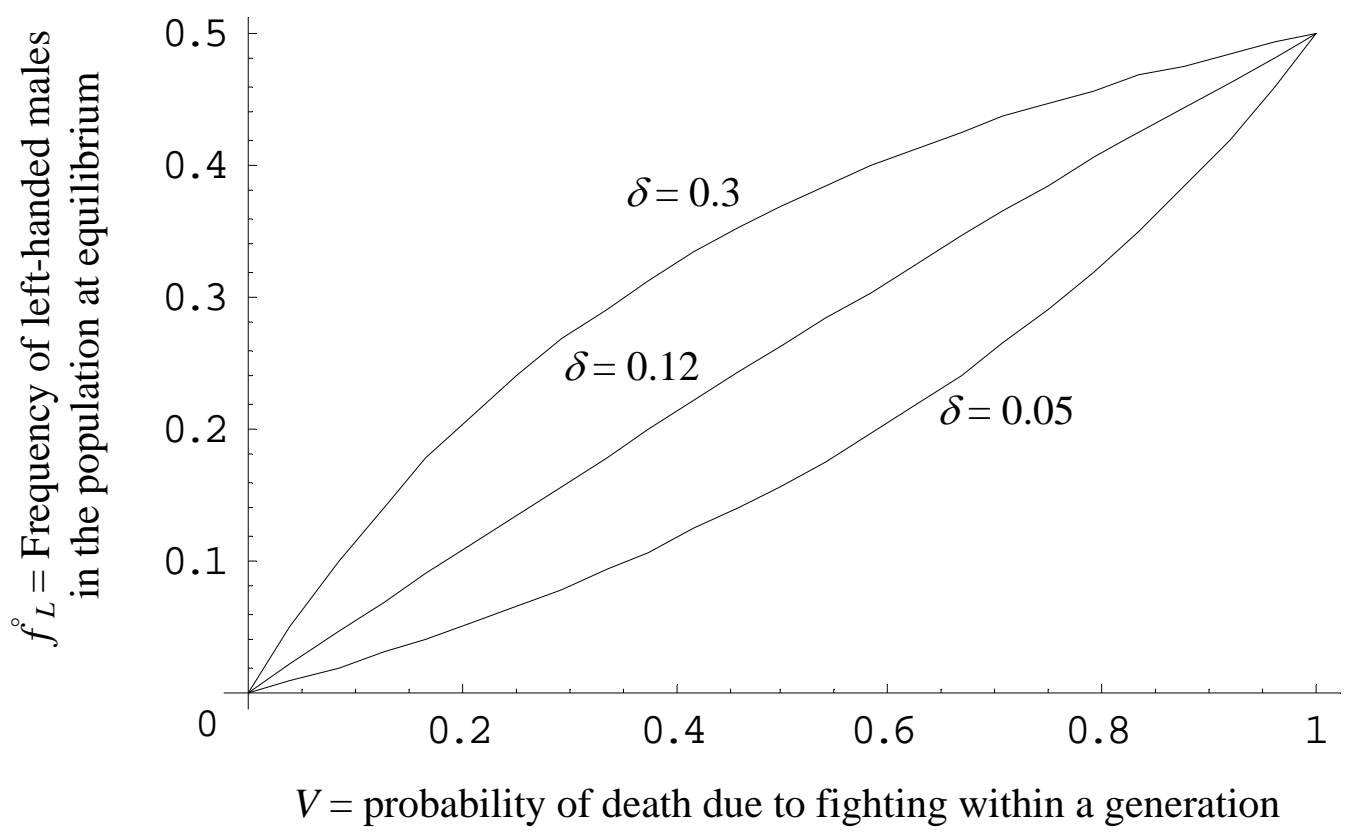


Figure 3. Frequency of left-handers at equilibrium as a function of their advantage in fights.

Effects of the social status advantage associated to fighting. For all curves $c=c_{\text {fight }}=0.1$,

except for the thick line for which $c=0.1$ and $c_{\text {fght }}=0.5 ; V=0.1$.

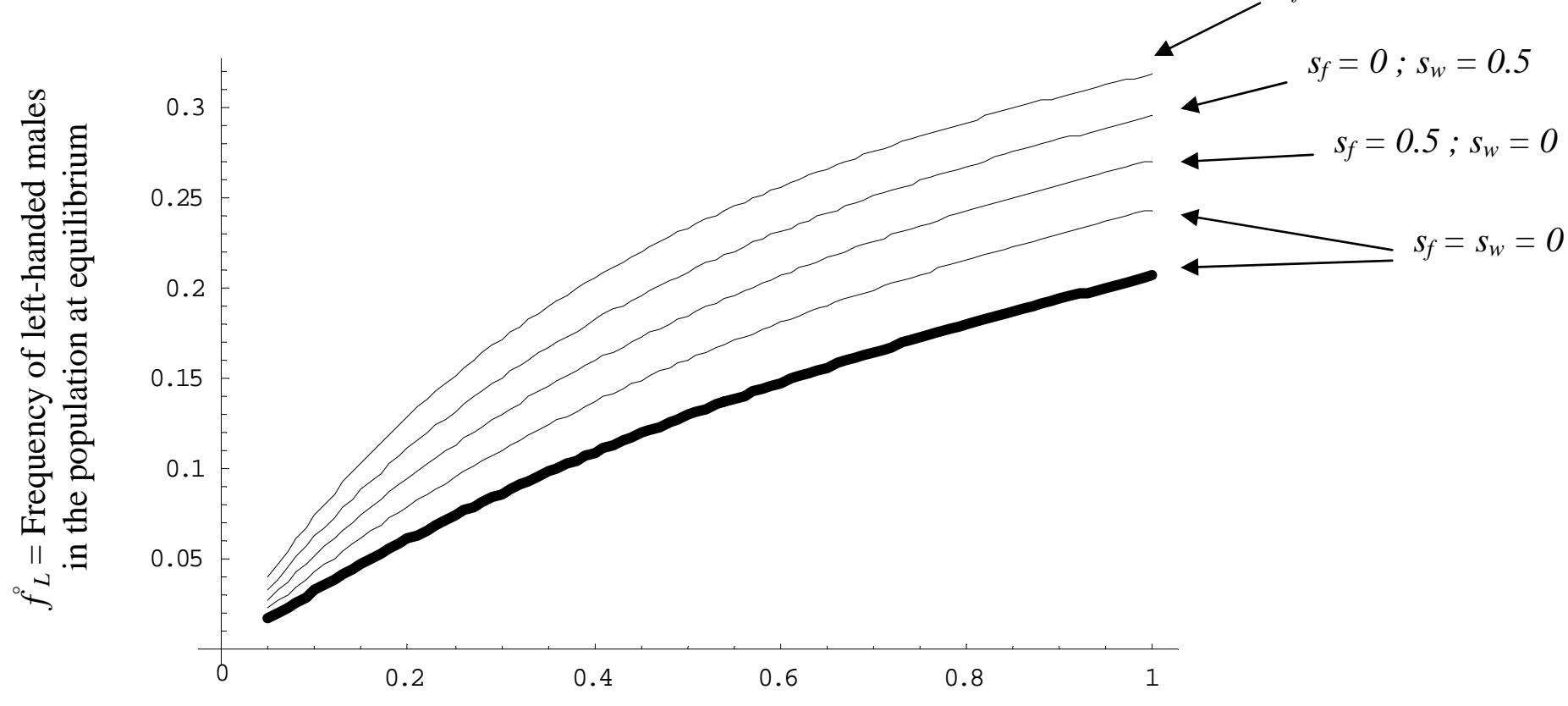

$\delta=$ Modulator of the advantage of left-handed males

Figure 4: Frequency of left-handers at equilibrium as a function of the advantage of lefthanded males. For all curves $p=q=\alpha_{\mathrm{M}}=\alpha_{\mathrm{F}}=\beta_{\mathrm{M}}=\beta_{\mathrm{F}}=0.1 ; c_{M}=c_{\mathrm{F}}=0.0$ for thin lines and $c_{M}=c_{F}=0.5$ for thick lines. Note that for $\delta=0$, the frequency of the left-handed males and females at equilibrium are the same because probability of transmission and cost are identical for both sexes.

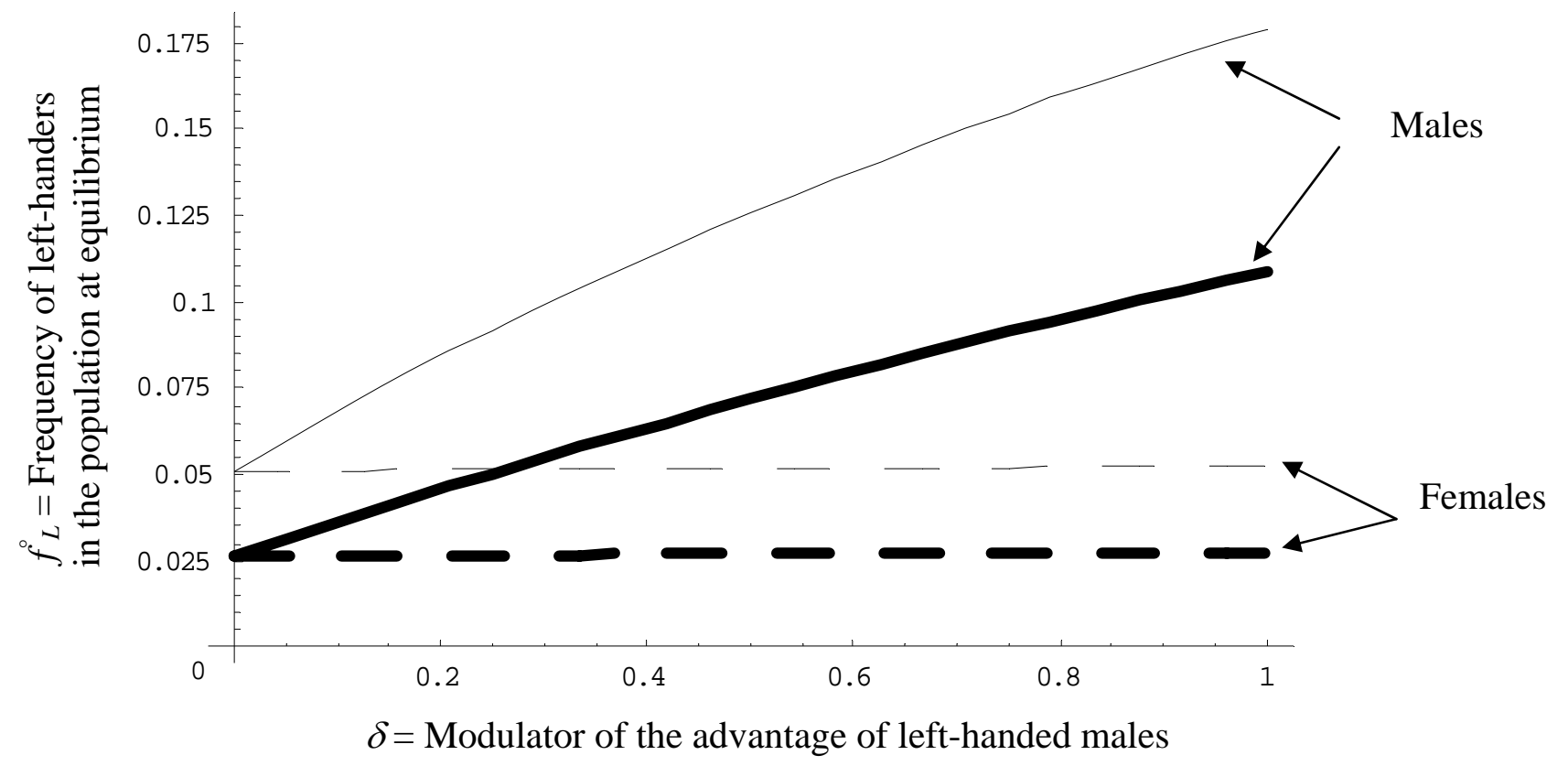


Figure 5. Frequency of left-handed females at equilibrium as a function of the advantage of males: the indirect advantage. For all curves $p=0.2 ; q=0.1 ; \alpha_{\mathrm{M}}=\alpha_{\mathrm{F}}=10^{-6} ; c_{m}=c_{f}=0.1$. For the thin line $\beta_{\mathrm{F}}=\beta_{\mathrm{M}}=0.1$ and for the thick line $\beta_{\mathrm{F}}=\beta_{\mathrm{M}}=1$.

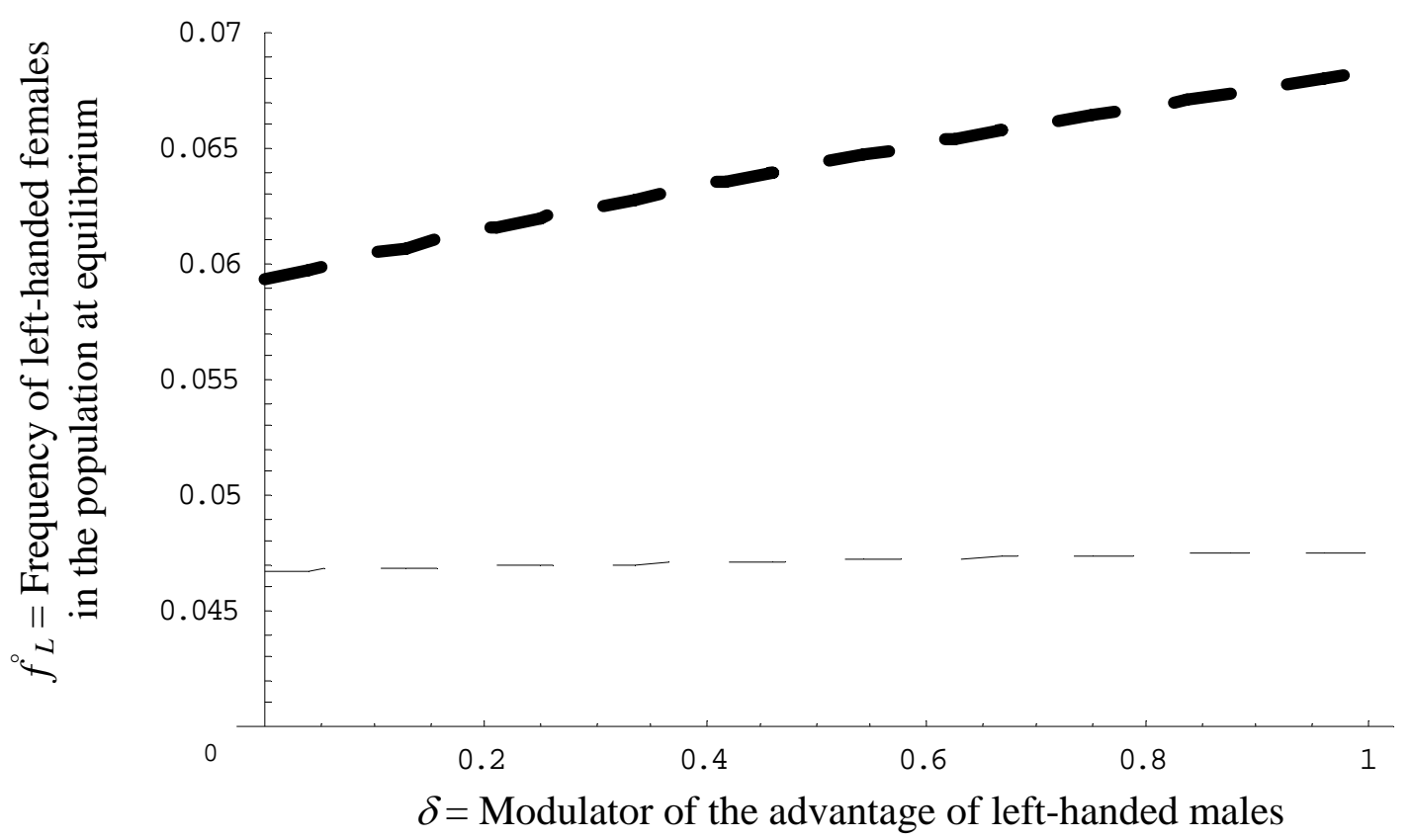

